\title{
Characterization of Rectifying and Sphere Curves in $\mathbb{R}^{3}$
}

Julie Logan and Yun Myung $O b^{*}$

Department of Mathematics, Andrews University, Berrien Springs, MI

Student:julie@andrews.edu

Mentor:oby@andrews.edu

\begin{abstract}
Studies of curves in 3D-space have been developed by many geometers and it is known that any regular curve in 3D space is completely determined by its curvature and torsion, up to position. Many results have been found to characterize various types of space curves in terms of conditions on the ratio of torsion to curvature. Under an extra condition on the constant curvature, Y. L. Seo and Y. M. Oh found the series solution when the ratio of torsion to curvature is a linear function. Furthermore, this solution is known to be a rectifying curve by B. Y. Chen's work. This project, uses a different approach to characterize these rectifying curves.

This paper investigates two problems. The first problem relates to figuring out what we can say about a unit speed curve with nonzero curvature if every rectifying plane of the curve passes through a fixed point $x_{0}$ in $\mathbb{R}^{3}$. Secondly, some formulas of curvature and torsion for sphere curves are identified.

KEYWORDS

Space Curve; Rectifying Curve; Curvature; Torsion; Rectifying Plane; Tangent Vector; Normal Vector; Binormal Vector
\end{abstract}

\section{INTRODUCTION}

Consider a unit speed curve $\alpha: I \rightarrow \mathbb{R}^{3}$, where $I=(a, b)$ is an interval on the real number line. Since it is a unit speed curve (i.e. $\alpha^{\prime}(s)$ has magnitude 1$)$, the unit tangent vector is $T(s)=\alpha^{\prime}(s)$.

Definition 1. The curvature, $\kappa(s)$, of a unit speed curve, $\alpha$, is defined as

$$
\kappa=\kappa(s)=\left|T^{\prime}(s)\right| \text {. }
$$

Equation 1.

The principal normal vector, $N(s)$, is defined by dividing $T^{\prime}(s)$ by its magnitude:

$$
N(s)=\frac{T^{\prime}(s)}{\left|T^{\prime}(s)\right|}=\frac{T^{\prime}(s)}{\kappa(s)} .
$$

Equation 2.

We then have $T^{\prime}(s)=\kappa(s) N(s)$. The binormal vector which is perpendicular to both $T(s)$ and $N(s)$ is defined as $B(s)=T(s) \times N(s)$.

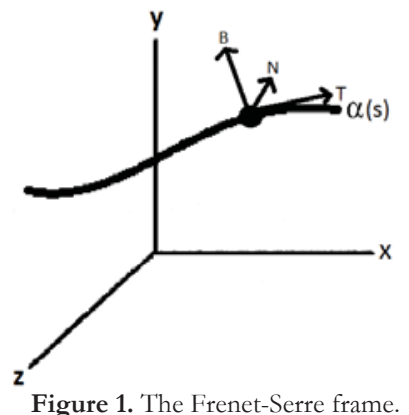

These three vectors $\{T(s), N(s), B(s)\}$ form an orthonormal basis in $\mathbb{R}^{3}$ along the curve which is called the FrenetSerre frame (Figure 1). So,

$$
B^{\prime}(s)=a(s) T(s)+b(s) N(s)+c(s) B(s)
$$

Equation 3.

for functions $a, b$, and $c$. It is easy to see that $c$ is zero from the fact that $B$ is a unit vector field. The fact that $a$ is zero follows from the perpendicularity of $T$ and $B$ together with the fact that $T^{\prime} \cdot B=0$, which follows from $T^{\prime}(s)=$ $\kappa(s) N(s)$. We are ready to define the torsion of the curve.

Definition 2. The torsion, $\tau(s)$, of the curve, $\alpha(s)$, is defined by the equation $B^{\prime}(s)=-\tau(s) N(s)$.

The curvature measures the deviation of a curve from being a line and torsion measures the deviation of a curve from being contained in a single plane.

Since we know that $T(s), N(s)$, and $B(s)$ are all mutually perpendicular to each other, we have $N(s)=B(s) \times T(s)$. Using the facts that $T^{\prime}(s)=\kappa(s) N(s)$ and $B^{\prime}(s)=-\tau(s) N(s)$ we derive the following: 


$$
\begin{aligned}
N^{\prime}(s) & =B^{\prime}(s) \times T(s)+B(s) T^{\prime}(s) \\
& =-\tau(s) N(s) \times T(s)+B(s) \times \kappa(s) N(s) \\
& =-\kappa(s) T(s)+\tau(s) B(s) .
\end{aligned}
$$

Equation 4.

Thus, we have the following Frenet-Serret formula:

$$
\begin{aligned}
T^{\prime} & =\kappa(s) N(s) \\
N^{\prime} & =-\kappa(s) T(s)+\tau(s) B(s) \\
B^{\prime} & =-\tau(s) N(s)
\end{aligned}
$$

Equation 5.

According to the Fundamental Theorem of Curves, any regular curve in $3 \mathrm{D}$ space is completely determined by its curvature and torsion, up to position. Several characterization facts have been found over the years. We know that any curve with constant curvature and zero torsion is a circle and the curve with a constant ratio of torsion to curvature is known to be a general helix.

Now, we need to introduce three types of planes along the curve. The osculating plane to a unit speed curve $\alpha(s)$ is the plane perpendicular to $B(s)$, the normal plane of $\alpha(s)$ is the plane perpendicular to $T(s)$ and the rectifying plane of $\alpha(s)$ is the plane perpendicular to $N(s)$ (Figure 2).

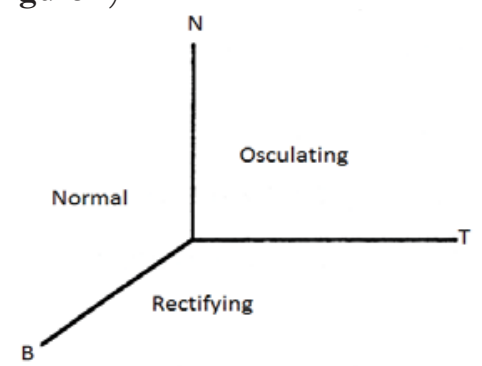

Figure 2. The normal, osculating and rectifying planes.

It has been shown that if every normal plane to the curve $\alpha(s)$ passes through a fixed point $x_{0}$ in $\mathbb{R}^{3}$, then the curve lies on a sphere, and if every osculating plane to the curve $\alpha(s)$ passes through a fixed point $x_{0}$ in $\mathbb{R}^{3}$, then the curve lies on a plane. ${ }^{1}$ Thus, it is natural to investigate the case where every rectifying plane goes through a given point $x_{0}$.

Definition 3. A rectifying curve is a space curve whose position vector lies in its rectifying plane. ${ }^{3}$

The idea of rectifying curves was introduced by B. Y. Chen and he provided many fundamental properties of the curves together with classification results. ${ }^{3}$

For the second problem investigated in this paper, we will need the following fact about the curvature and torsion of "sphere curves", i.e. curves in $\mathbb{R}^{3}$ lying on a sphere.

Proposition: If $\alpha(s)$ is a unit speed curve with $\kappa \neq 0, \tau \neq 0$, then $\alpha(s)$ lies on a sphere if and only if

$$
\frac{\tau}{\kappa}=\left(\frac{\kappa^{\prime}}{\tau \kappa^{2}}\right)^{\prime} \text {. }
$$

Equation $6 .^{1}$

\section{RESULTS}

The first problem we worked on was finding a necessary condition for a unit speed curve if every rectifying plane contains the point $x_{0}$ in $\mathbb{R}^{3}$. Since the rectifying plane is orthogonal to $N$, we have $\left(\alpha(s)-x_{0}\right) \cdot N=0$. Thus, taking the derivative of both sides we find

$$
0=\alpha^{\prime}(s) \cdot N+\left(\alpha(s)-x_{0}\right) \cdot N^{\prime}
$$

Equation 7.

Then by substituting from the Frenet-Serret formula we have

$$
\begin{aligned}
0 & =T \cdot N+\left(\alpha(s)-x_{0}\right) \cdot(-\kappa(s) T(s)+\tau(s) B(s)) \\
& =\left(\alpha(s)-x_{0}\right) \cdot(-\kappa(s) T(s)+\tau(s) B(s))
\end{aligned}
$$

Equation 8.

since $T \cdot N=0$. We know

$$
0=-\kappa(s)\left(\alpha(s)-x_{0}\right) \cdot T(s)+\tau(s)\left(\alpha(s)-x_{0}\right) \cdot B(s) . \quad \text { Equation } 9 .
$$

Thus,

$$
\kappa(s)\left(\alpha(s)-x_{0}\right) \cdot T(s)=\tau(s)\left(\alpha(s)-x_{0}\right) \cdot B(s) .
$$

Equation 10.

Then, taking another derivative of Equation 8, we obtain

$$
\begin{aligned}
& 0=\left[\left(\alpha(s)-x_{0}\right) \cdot(-\kappa(s) T(s)+\tau(s) B(s))\right]^{\prime} \\
& =T(s) \cdot(-\kappa(s) T(s)+\tau(s) B(s))+\left(\alpha(s)-x_{0}\right) \cdot\left(-\kappa^{\prime}(s) T(s)-\kappa(s) T^{\prime}(s)+\tau^{\prime}(s) B(s)+\tau(s) B^{\prime}(s)\right) \\
& =-\kappa(s)+\left(\alpha(s)-x_{0}\right) \cdot\left(-\kappa^{\prime}(s) T(s)-\kappa^{2}(s) N(s)+\tau^{\prime}(s) B(s)-\tau^{2}(s) N(s)\right) \\
& =-\kappa(s)-\kappa^{\prime}(s)\left(\alpha(s)-x_{0}\right) \cdot T(s)-\kappa^{2}(s)\left(\alpha(s)-x_{0}\right) \cdot N(s)+\tau^{\prime}(s)\left(\alpha(s)-x_{0}\right) \cdot B(s)-\tau^{2}(s)\left(\alpha(s)-x_{0}\right) \cdot N(s) \\
& =-\kappa(s)-\kappa^{\prime}(s)\left(\alpha(s)-x_{0}\right) \cdot T(s)+\tau^{\prime}(s)\left(\alpha(s)-x_{0}\right) \cdot B(s) .
\end{aligned}
$$


So by Equation 10, we have $\kappa(s)=-\kappa^{\prime}(s)\left(\alpha(s)-x_{0}\right) \cdot T(s)+\tau^{\prime}(s)\left(\frac{\kappa(s)}{\tau(s)}\right)\left(\alpha(s)-x_{0}\right) \cdot T(s)$ and by simplification we obtain

Therefore,

$$
\kappa(s)=\left(\alpha(s)-x_{0}\right) \cdot T(s)\left[-\kappa^{\prime}(s)+\tau^{\prime}(s)\left(\frac{\kappa(s)}{\tau(s)}\right)\right] .
$$

$$
\begin{gathered}
\left(\alpha(s)-x_{0}\right) \cdot T(s)=\frac{\kappa(s)}{-\kappa^{\prime}(s)+\tau^{\prime}(s)\left(\frac{\kappa(s)}{\tau(s)}\right)} \\
\left(\alpha(s)-x_{0}\right) \cdot B(s)=\frac{\kappa(s)}{\tau(s)} \frac{\kappa(s)}{-\kappa^{\prime}(s)+\tau^{\prime}(s)\left(\frac{\kappa(s)}{\tau(s)}\right)} .
\end{gathered}
$$

Then working with the denominator of Equation 13,

$$
\begin{aligned}
-\kappa^{\prime}(s)+\tau^{\prime}(s)\left(\frac{\kappa(s)}{\tau(s)}\right) & =\frac{\tau^{\prime}(s) \kappa(s)-\kappa^{\prime}(s) \tau(s)}{\tau(s)} \\
& =\frac{\left(\frac{\tau(s)}{\kappa(s)}\right)^{\prime} \kappa^{2}(s)}{\tau(s)} .
\end{aligned}
$$

Equation 15.

Equation 13 becomes

$$
\begin{aligned}
\left(\alpha(s)-x_{0}\right) \cdot T(s) & =\frac{\kappa(s)}{-\kappa^{\prime}(s)+\tau^{\prime}(s)\left(\frac{\kappa(s)}{\tau(s)}\right)} \\
& =\frac{\tau(s)}{\left(\frac{\tau(s)}{\kappa(s)}\right)^{\prime} \kappa(s)} \\
& =\frac{\frac{\tau(s)}{\kappa(s)}}{\left(\frac{\tau(s)}{\kappa(s)}\right)^{\top}} .
\end{aligned}
$$

Equation 16.

In a similar manner, since we know that $\left(\alpha(s)-x_{0}\right) \cdot B(s)=\frac{\kappa(s)}{\tau(s)}\left(\alpha(s)-x_{0}\right) \cdot T(s)$,

$$
\left(\alpha(s)-x_{0}\right) \cdot B(s)=\frac{1}{\left(\frac{\tau(s)}{\kappa(s)}\right)^{\prime}} .
$$

Equation 17.

Therefore

$$
\alpha(s)-x_{0}=\frac{\frac{\tau(s)}{\kappa(s)}}{\left(\frac{\tau(s)}{\kappa(s)}\right)^{\prime}} T(s)+\frac{1}{\left(\frac{\tau(s)}{\kappa(s)}\right)^{\prime}} B(s) .
$$

Equation 18.

We then let $t(s)=\frac{\tau(s)}{\kappa(s)}$. Equation 18 becomes

$$
\alpha(s)-x_{0}=\frac{t(s)}{t^{\prime}(s)} T(s)+\frac{1}{t^{\prime}(s)} B(s) .
$$

Equation 19.

We take another derivative of Equation 18, to find

$$
\left(\alpha(s)-x_{0}\right)^{\prime}=\left(\frac{t(s)}{t^{\prime}(s)}\right)^{\prime} T(s)+\left(\frac{t(s)}{t^{\prime}(s)}\right) T^{\prime}(s)+\left(\frac{1}{t^{\prime}(s)}\right)^{\prime} B(s)+\left(\frac{1}{t^{\prime}(s)}\right) B^{\prime}(s)
$$

Equation 20.

and since $\left(\alpha(s)-x_{0}\right)^{\prime}=T(s)$,

We then obtain

$$
T(s)=\left(\frac{t(s)}{t^{\prime}(s)}\right)^{\prime} T(s)+\left(\frac{t(s)}{t^{\prime}(s)}\right) \kappa(s) N(s)+\left(\frac{1}{t^{\prime}(s)}\right)^{\prime} B(s)-\left(\frac{1}{t^{\prime}(s)}\right) \tau(s) N(s) . \quad \text { Equation } 21 .
$$

$$
0=\left(-1+\left(\frac{t(s)}{t^{\prime}(s)}\right)^{\prime}\right) T(s)+\left(\frac{\kappa(s) t(s)-\tau(s)}{t^{\prime}(s)}\right) N(s)+\left(\frac{1}{t^{\prime}(s)}\right)^{\prime} B(s)
$$

Equation 22.

and we now note that $T, N$ and $B$ are linearly independent. Thus,

$$
\begin{gathered}
0=-1+\left(\frac{t(s)}{t^{\prime}(s)}\right)^{\prime} \\
0=\left(\frac{1}{t^{\prime}(s)}\right)^{\prime} .
\end{gathered}
$$

Then, working with Equation 24, we have $t^{\prime \prime}(s)=0, t^{\prime}(s)=c$ and $t(s)=c s+d$ for some constants $c, d$ and arc length $s$. This solution satisfies Equation 23 as well.

Here is the summary of the first result:

Theorem A: Suppose $\alpha$ is a unit speed curve with nonzero curvature.

If every rectifying plane contains the point $x_{0}$ in $\mathbb{R}^{3}$, i.e., if $\alpha$ is a rectifying curve, then its ratio $\tau / \kappa$ is a linear function.

Note: This result was obtained by B. Y. Chen in Theorem $2,{ }^{3}$ but it was derived here by a different method.

Our next task is to get the formula for the curvature and torsion for a sphere curve. From the Proposition,

$\alpha$ is a sphere curve in $\mathbb{R}^{3}$ if and only if $\frac{\tau}{\kappa}=\left(\frac{\kappa^{\prime}}{\tau \kappa^{2}}\right)^{\prime}$. Let $f(s)=\frac{\kappa^{\prime}}{\tau \kappa^{2}}$. Then $\tau=f^{\prime}(s) \kappa$. Thus,

$$
\frac{\kappa^{\prime}}{2}=\tau f(s) \text {. }
$$

Equation 25. 
Substituting $\tau=f^{\prime}(s) \kappa$,

which yields

$$
\frac{\kappa^{\prime}}{\kappa^{2}}=f^{\prime}(s) \kappa f(s) \quad \text { Equation } 26 .
$$

By rearranging the terms

$$
\begin{aligned}
& \kappa^{\prime}=\frac{d \kappa}{d s} \\
& =f^{\prime}(s) \kappa^{3} f(s) . \quad \text { Equation } 27 . \\
& \frac{d \kappa}{\kappa^{3}}=f^{\prime}(s) f(s) d s .
\end{aligned}
$$

Taking the integral of both sides to solve the separable differential equation we obtain $\frac{-1}{2 \kappa^{2}}=\frac{1}{2} f(s)^{2}+c_{0}$ for a constant $c_{0}$ and then $\frac{1}{\kappa^{2}}=-f(s)^{2}+c$ for a constant $c=-2 c_{0}$.

By solving for $\kappa$, we find

$$
\kappa=\frac{1}{\sqrt{c-f(s)^{2}}}
$$

Equation 29.

and since we know that $\tau=f^{\prime}(s) \kappa$, we use this formula for $\kappa$ to get

$$
\tau=\frac{f^{\prime}(s)}{\sqrt{c-f(s)^{2}}}
$$

Equation 30.

for a differentiable function $f(s)$ and a constant $c=-2 c_{0}>0$.

The above result can be summarized as follows:

Theorem B: For a sphere curve in $\mathbb{R}^{3}$, its curvature $\kappa$ and $\tau$ are given by

$$
\kappa=\frac{1}{\sqrt{c-f(s)^{2}}} \text { and } \tau=\frac{f^{\prime}(s)}{\sqrt{c-f(s)^{2}}}
$$

Equation 31.

for a constant $c>0$ and a differentiable function $f(s)$.

\section{DISCUSSION}

Centrodes are a particular kind of rectifying curve and they are useful in mechanics and joint kinematics. ${ }^{4}$ Another example of application appeared in the Journal of Hand Surgery. ${ }^{5}$

Using the formulas for curvature and torsion of sphere curves we have found, future work would include finding a formula for the original sphere curve satisfying given curvature and torsion with specific $c$ and differentiable function $f(s)$. This is a continuation of work by Ye Lim Seo and Yun Myung Oh published in American Journal of

Undergraduate Research in Jan, $2015 .^{6}$

\section{REFERENCES}

1. Millman, R. and Parker, G. (1977) Elements of differential geometry, Prentice Hall, Englewood Cliffs, N. J. , 33-37

2. Curvature, Torsion and the Frenet Frame. blogimages.bloggen.be/gnomon/attach/203774.pdf.

3. Chen, B. Y. (2003) When Does the Position Vector of a Space Curve Always Lie in Its Rectifying Plane?, Amer. Math. Monthly 110, 147-152.

4. Chen, B. Y. and Dillen, F. (2005) Rectifying Curves as Centrodes and Extremal Curves, Bulletin of the Institute of Mathematics Academia Sinica 33 No. 2, 77-90.

5. Weiler, P.J. and Bogoch, R. E. (1995) Kinematics of the distal radioulnar joint in rheumatoid-arthritis-an in-vivo study using centrode analysis, J. Hand Surgery 20A, 937-943.

6. Oh, Y. M. and Seo, Y. L. (2015) A Curve Satisfying $\tau / \kappa=s$ with constant $\kappa>0$, American Journal of Undergraduate Research 12, 57-62.

\section{ABOUT THE STUDENT AUTHOR}

Julie Logan is an undergraduate student at Andrews University and this paper is a requirement of the honors program at Andrews University. She has interest in mathematics and has earned several awards for excellence in mathematics classes. She is planning to apply for graduate work in mathematics.

\section{PRESS SUMMARY}

It is known that for every rectifying curve, the ratio $\tau / \kappa$ is a linear function. In this paper, we give a different proof of the result. Besides, we also derive formulas for the curvature and the torsion of sphere curves as well.

\section{ACKNOWLEDGEMENTS}

The authors thank the Andrews University Research Office and J. N. Andrews Honors program for assistant through the 2015-2016 Undergraduate Research Assistant Scholarship. The authors are very grateful to the referees for several valuable suggestions. 